\title{
LE ROMAN DU SAUMON
}

Par M. R. de DROUIN de BOUVILle

(Suite) (1)

M. I.ıssoine, Abbé de la Val-Dieu, au confluent de la Neuse et de la Semois, où existe une importante pêcherie sur cette dernière rivière, rapporte, qu'au dire des pêcheurs : "quand il n'y a point de débordement, comme il ne passe point de Saumons au-dessus de la pêcherie, la pêche d:s Saumoneaux, qu'ils appellent "Gillons ", manque le printemps suivant ".

M. D’ArṬus, Major d'inlanterie, Ingénieur en chef à IIuningue, relate que " suivant nos pêcheurs riverains, il est constant que, vers la fin de Novembre, les Saumons femelles viennent jeter leurs oufs dans des espéces de fosses qu'elles creusent ì dessein ì l'embouchure de la Veissen el que les Saumons mâles les fécoudent en les arrosant de leur laitance, que la chaleur du printemps les fitit éclore et, qu'alors, cette fartie de rivière foisonne de Saumonneaux ou petits Saumons.

"On remarque que les femelles de ces Saumonneaur n'ont pas d'ceufs dans le corps et que les mâles onl de la laitance. It y en a qui prétendent que ces petits mâles frayent avec les femelles adultes "...

"L'instinct engage les Saumonneaux à retourner à la mer où ils piennent leur croissance ef reviennent dans les rivières pour travailler à leur propagation. "

Finalement Dufamsi se juge sulfìsammend informé pour opiner :

"Laissant à part les systèmes pour nous en tenir aux fails les mieux constatés, je crois que les Saumous remontent dans les rivières pour $y$ Irayer et y déposer leurs orufs" ( 2 ).

Conclusion modeste, comme insimuée, mais la suggestion vient au terme d'une dissertation trop fournie, trop bien conduite pour n'être pas convaincante. Aussi doit-on reconnaître à Dunsmfi, re nérite, s'ajoutant à bien d'autres, d'avoir établi de façon péremptoire, que la migration du Saumon adulte est un voyage nuplial, que la reproduction s'effectue aux lêtes de bassins et que les Tocans sont bien de petits Samons qui, parvemus à un certain degré de développement, dóvalent pour aller s'engraisser ('n mer.

Mentionnons encore, avant de quitter Dumanri., qu'il cst le premier à avoir mis en relief les influences qui régissent la remonte des Saumons :

(s) Voir Bulletin, Septembre rgag, p. 54 ; Novembre rgag, p. 103 ; Mars rg3o, p. 203.

(2) Tout on posant colte rìnle, Dthavri. admet qu'elle pellt rompiorter exception : "Je crois bien qu'il y a quelques Saumons qui frayent à la mer, puisqu'on prend de petits Saumoneaux dans les pares". 
"Ils se plaisent dans les eaux vives et claires qui coulent sur un fond de sable ou de gravier... Souvent, après avoir remonté dans une grande rivière un espace assez considérable, ils la quittent pour passer dans un ruisseiu dont l'ea! est plus vive" .

Le Seigneur du Monccau est aussi le premier à avoir donné une description détaillẻe, accompaşnée de remarquables figures, du parasite qui, selon l'opinion du temps, contraingait le Satumon a quitter l'Océan, car : "d'autres prétendent cut'ils n culrent dans les rivières que pour se débar. rasser des Insectes dont nous avons lẹ́jà partlé et qu'ils sont engagés à retourner à la mer pur se guérir des maladies qu'ils contractent dans l'eau douce $n$.

Cet Insecte est un petil (irustace, qui it reçu, depuis, le nom de lemaea salmonea.

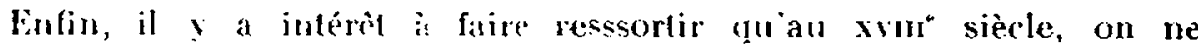
croi fruère an retour rlés siumuns sur les frayères où ils sont éclos. "Plusieurs prétendent quils rentrent dins les mêmes rivières où ils ont pris naissarice " A ret extrail dine lellue de M. n'Inrus, se borne la placé arcorlée, par le Traile des peseftes, ì lénoncé d'une hypothèse

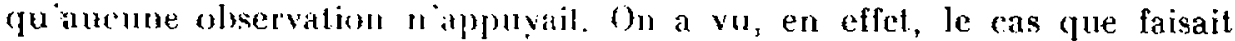
notre autur des essais de marufuacre de: 'Tocans effectués de son temps à Châteaulin ou ailleurs.

Des expériences de Brelagrne, mons savons, d'ailleus, par Marc-Elièzec ficocir (1 $723-1799$ ), auleur diuse imposante lehthyologie (a) en r6 volumes in-folio, dont 1.2 de discours et 4 de planches, dédiée à la reine Marie-Antoinelfe, quelles porlèreat sculemenl sur une douzaine d'indi. vidus. Or : - " daus da suite less procheurs... ont assuró que, la première année, ils avaient pris cing de ces Saumons marqués, la seconde annét encore trois el la troisjèn e annié aussi- trois " Le compte y était donc, à line unité près ; on comprend que Dumantu, se soit méfié.

Le naturaliste alemand, moins prudent, s'extasie : - " Une chose remarquable est que le Poisson retronve l'endroit où il a frayé comme lHirondelle le bâtiment où elle a lait sont nid" (2).

Remarcuons quil ne s'agrirait plus, alors, d'un retour de l'animal arhulte au lieu de sa naissance, mais à relui des premières amours. La fidélité à leur souvenir, présidant aux migrations du Poisson comme à relles de l'Oiseau, il y arat la de quoi charmer les cocurs sensibles, nom breux à l'époque. Btoch, tout mésfecin qu'il fut et homme de science, étail de son temps et avait un culte attendri et admiratif de la nature.

"On voit aussi, - dit-il - par Ie Saumon, la force du penchant qu'ont les animaux purt 'a propusation de leur espèce. Il abandonne les

t-: Ichthyologie ou Histoire naturelle gênérale el particulière des Poissons, -.: Berlin, $1-85-1-87$.

(3) Il s'agit d'unc citation tirée tu Cours d'histoire naturelse, tome $V, p .293 ;-$ Paris, $17 \%$; - mais Block jułrait bien faire sienne, sans d'ailleurs en fournir d'autro. justification que les marquages de Châtemlin, l'opintion émise. 
mers qui fourmillent d'aminaux el où il trouve une nourriture abondante, va dans les fleuves que les Hommes dépeuplent, tâche, par toutes sortes de ruses, d'échapper au pécheur, surmonte mille obstacles livers au cuurs de son long voyage ct tout cela pour trouver un endroit soummole où il puisse déposer ses ceufs".

Par ailleurs, Bıocr semble bien ille lichthyologiste qui, à l'encontre de l'opinion de Dunames, a fail privaloir celle que tous les Bécaris blajent du même sexe.

"Dans les males qui ant pris tont lour accoissement, la michrire iuférieure forme un crochet émoussé qui semboite dans un enfoncement de la màchoire supérieure"

A cette indication donmés par le premicr volume, il ajoute dans le troi sjème

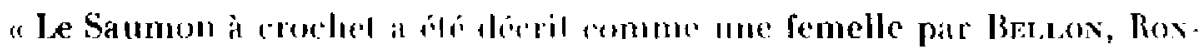

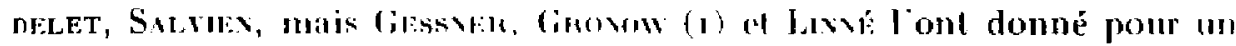

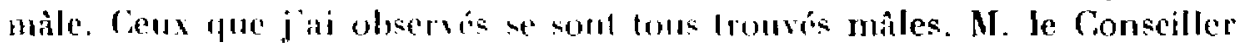

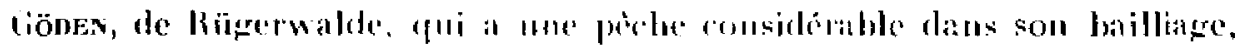
me marque que, de plusidurs milliegs de ces Poissons que ses grens ont ruverts pour les fumcer el envoyer dehors, il ne s'est pas trouvé une seule firmelle gui eut un crochet ".

Il n'est pas imutile rle noter qu: liügremualde csl une ville còtière de la Poméranie, à lembourluture d'un pelil tribulaire de la Baltique, le Wipper. I.es observalions qui ? lurent liniles russeul demandé vérification; on

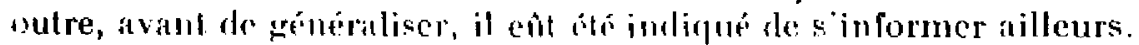

La référence de Buocal à laxví amène à anc constalation méritant mentlion.

En se reportant an lexle de la dixième édition, révisée par l'auteur, du célèbre Systema nulurre (a) on trowve ce qui suit :

Trulle compore variegalo.

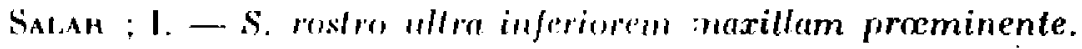

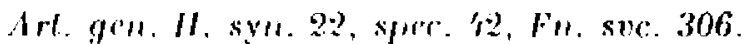

Habilat in Oceomo Europas, parit in fluvis.

B. $12-$ D. $15-13.1 / 1-1.11-\Lambda .13-$ C. 19 .

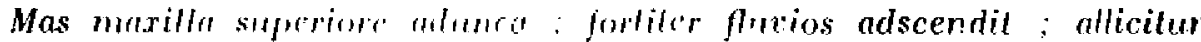
rilbo.

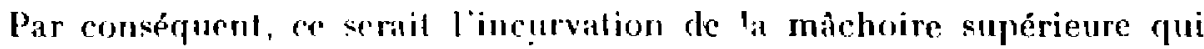
serait surtuut remarquablè ; sans doule y a-t-il là un lapsus calami ou une inute d'impression.

On peut s'étonner aussi de voir signaler, par un naturaliste aussi concis

(1) Museum iclhyologirum, sistens Piscium indigenorum ct quorundam exollicorum descriptiones, - Lughuni Bafmorum, 1-5/-т,56.

(2) Caroli Linval:t. Syslema Natura per tria regna nalaru, secundam classes, ordines, genera, spacies, - editio decima, reformala. - Holmia, $17^{58 .}$ 
que Lınné, que le Saumon serait altiré par la couleur blanche. Mais il était Suédois et il s'agit d'une croyanoe de pêcheurs scandinaves dont le pre. mier auteur qui ait parlé paraît avoir été le pasteur danois Eric Ponro. prdDan ( $698-1764)$, dit le Jeune (1).

Dunamel en dit ce qui suit, avec planche á l'appui :

"Voici encore une autre manière assez singulièrc de pôcher le Saumon in Norvège.

"Comme les pêcheurs savent que les Saumons sont attirés par les; c bjets blancs, soit qu'ils les prennent pour des monceaux de neige' d'où coule de l'eau très fraîche qui leur plaît, soit, comme on le pense com. munément, qu'ils prennent ces endroits blancs pour des nappes d'ean qu'ils cherchent, comme l'on sait, par dessus toutes choses, les habitanls choisissent un endroit où les rochers forment un espèce de cul-de-sac... qui ail sept à huit brasses de profondeur ct où se rende un courant d'eau ; ils blanchissent avec de la chaux les rochers qui sont au fond de ce cul de-sac et ils tendent un filet près de l'critréc ; ils le laissent tomber au fond de l'eau ; puis, étant cachés dans un repli de rochers ils observent quand il entre des Poissons dans cet enfoncement ; lorsqu'ils en aper çoivent, ils halent sur une corde pour élever le filet et le tendre par Ir travers de l'embouchure de cecul-de-sac afin d'empecher le Poisson d'en sortir ".

On peut se demander si ce procédé "singulier " était efficace et Lrvoú bien informé.

Quoi qu'il en soit, nous voici à la fin du xvu' siècle.

On voit ce qu'on connaissait alors du Saumon ; certains points de son histoire étaient élucidés, grâce à de récentes observations, mais que de mystères encore qui devaient tenir la science en échec durant une centaine d'années ! Ceci comme conséguence de la Révolution qui, on ie verra, amena l'oubli rapide des travaux de Dumamel nu Monceau et de ses contemporains.

\section{CONSULTATIONS TECHNIQUES}

8) D. - Existe-t-il des élangs où soit pratiqued l'astacicullure ? - V., à R. (Indre).

R. - On trouve quelquefois dans les étangs l'Wcrevisse a patles rouges, mais il nc semble pas qu'il soit procédé, quelque part en France, à un élesage méthodique de ce. Crustace.

Son introduction dans les eaux nù il niexiste pas naturollement est rarement couronnée de succès. En tout cas, si on vent tenter III essai, le micux est d'immerger

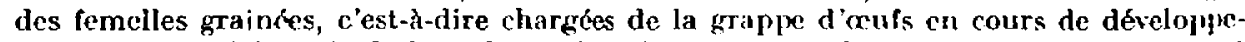
ment. Il faut s'abstenir de lis acheter dans le commerce, les sujets qu'il fournit ayant toutes chances d'etre malades ou porteurs de germes.

Prière de se reporter, pour le surplus, à la consultation insénée dans le Bulletin d'Octobre 1929, p. 80.

(1) Versuch einer natarlichen Historie von Norwegen. - Koppenhagen, 1753-1754. 